\title{
Final State Problem for the Dirac-Klein-Gordon Equations in Two Space Dimensions
}

\author{
Masahiro Ikeda \\ Department of Mathematics, Graduate School of Science, Osaka University, Toyonaka, Osaka 560 0043, Japan \\ Correspondence should be addressed to Masahiro Ikeda; m-ikeda@cr.math.sci.osaka-u.ac.jp
}

Received 25 April 2013; Accepted 15 July 2013

Academic Editor: Daniel C. Biles

Copyright (C) 2013 Masahiro Ikeda. This is an open access article distributed under the Creative Commons Attribution License, which permits unrestricted use, distribution, and reproduction in any medium, provided the original work is properly cited.

We study the final state problem for the Dirac-Klein-Gordon equations (DKG) in two space dimensions. We prove that if the nonresonance mass condition is satisfied, then the wave operator for DKG is well defined from a neighborhood at the origin in lower order weighted Sobolev space to some Sobolev space.

\section{Introduction}

We study the final state problem for the Dirac-Klein-Gordon equations (DKG) in two space dimensions:

$$
\begin{aligned}
& \left(\partial_{t}+\alpha \cdot \nabla+i M \beta\right) \psi=\phi \beta \psi, \\
& \left(\partial_{t}^{2}-\Delta+m^{2}\right) \phi=\psi^{*} \beta \psi, \quad(t, x) \in \mathbb{R} \times \mathbb{R}^{2},
\end{aligned}
$$

where $(\psi, \phi)$ is a $\mathbb{C}^{2} \times \mathbb{R}$-valued unknown function of $(t, x), \psi=\left(\psi_{1}, \psi_{2}\right)^{t}$ stands a spinor field and $\phi$ denotes a scalar field, $M, m>0$ denote masses of the spinor field and the scalar field, respectively, and $\psi^{*}$ denotes a transposed conjugate to $\psi$. The operators $\alpha \cdot \nabla$ and $\Delta$ are defined by $\alpha \cdot \nabla=\sum_{j=1}^{2} \alpha_{j} \partial_{x_{j}}$ and $\Delta=\sum_{j=1}^{2} \partial_{x_{j}}^{2}$, respectively. Here, $\alpha_{j}(j=1,2)$ and $\beta$ are Dirac matrices, that is, $2 \times 2$ selfadjoint matrices with constant elements such that

$$
\begin{gathered}
\alpha_{j}^{2}=\beta^{2}=I, \quad \alpha_{j} \beta+\beta \alpha_{j}=O, \quad \text { for } j=1,2, \\
\alpha_{j} \alpha_{k}+\alpha_{k} \alpha_{j}=O, \quad \text { for } j, k=1,2, \quad j \neq k .
\end{gathered}
$$

Our aim in the present paper is to show existence of the wave operator for the DKG system (DKG) under the nonresonance mass condition $m \neq 2 M$ in two space dimensions.

First, we recall some well-posedness results for (DKG). Many local well-posedness results in low-order Sobolev spaces have been obtained for these ten years (for recent information see, e.g., $[1,2]$ and references therein). Global well-posedness results in $2 \mathrm{~d}$ case were also obtained (see, e.g., [3]). Moreover, very recently, unconditional uniqueness in $2 \mathrm{~d}$ case was discussed in $[4,5]$. On the other hand, there are few results about scattering for (DKG) in $2 \mathrm{~d}$ case.

In $[6,7]$, the asymptotic behavior of solutions for DKG system was studied in $3 \mathrm{~d}$ case by reducing it to a nonlinear Klein-Gordon system (KG). Denote $\mathscr{D}_{ \pm} \equiv \partial_{t} \pm(\alpha \cdot \nabla+i M \beta)$. In view of the properties (1), we have

$$
\mathscr{D}_{-} \mathscr{D}_{+}=\partial_{t}^{2}-(\alpha \cdot \nabla+i M \beta)(\alpha \cdot \nabla+i M \beta)=\partial_{t}^{2}+\langle\nabla\rangle_{M}^{2} \text {, }
$$

where $\langle\nabla\rangle_{M} \equiv \sqrt{M^{2}-\Delta}$. Hence, multiplying both sides of the Dirac part by $\mathscr{D}_{-}$, we obtain

$$
\begin{aligned}
\left(\partial_{t}^{2}+\langle\nabla\rangle_{M}^{2}\right) \psi & =\mathscr{D}_{-}(\phi \beta \psi) \\
& =\left(\mathscr{D}_{-} \phi\right) \beta \psi-i M \phi I \psi+\phi \beta \mathscr{D}_{+} \psi \\
& =\left(\left(\mathscr{D}_{-} \phi\right) \beta-i M \phi I+\lambda \phi^{2} I\right) \psi,
\end{aligned}
$$

where we have used the fact that $\psi$ is the solution of the DKG system. Thus, the solution of the DKG system satisfies the following KG one:

$$
\begin{gathered}
\left(\partial_{t}^{2}+\langle\nabla\rangle_{M}^{2}\right) \psi=\left(\left(\mathscr{D}_{-} \phi\right) \beta-i M \phi I+\lambda \phi^{2} I\right) \psi \\
\left(\partial_{t}^{2}+\langle\nabla\rangle_{m}^{2}\right) \phi=\psi^{*} \beta \psi .
\end{gathered}
$$


If we want to obtain a priori estimates to the local solution for the DKG system, we can use estimates to solutions for the above KG one. Moreover, in the present two-dimensional case, the initial value problem for nonlinear KG systems including (4) was studied in [8] (see also [9]). In [8], Sunagawa proved existence of a unique global asymptotically free solution under the nonresonance mass conditions, if the initial data are sufficiently small, smooth and decay fast at infinity. However, asymptotic behavior of solutions for DKG is not clear because (DKG) is not equivalent to (4) in general. In this paper, we will consider the DKG system itself without reducing it into (4) such as in [10]. Though the initial value problem for DKG was treated in [11], the final value problem which will be discussed in this paper is more delicate because of the derivative loss difficulties.

In [10], the wave operator for the DKG system has been obtained in a three-dimensional case. They dealt with the DKG system itself. Nevertheless, from a point of time decay property for the free solutions of the DKG system, two dimensional-case is critical, that is, borderline case between the long range scattering and the short range one. Therefore, their argument cannot be applicable to the two-dimensional case. To overcome the lack of time decay property, we will use the algebraic normal form transformation developed in paper [8] and the decomposition of the Klein-Gordon operator, that is,

$$
\partial_{t}^{2}+\langle\nabla\rangle_{M}^{2}=\mathscr{D}_{+} \mathscr{D}_{-}
$$

By this combination, we will find a suitable second approximate solution to $\psi$ (given by (42)). We note that the implicit null structure for (DKG) was discovered in [12], and it was used to prove local well-posedness in low regular setting in [2]. On the other hand, in this paper, by explicit null structure, wave operator for (DKG) will be constructed.

Next, we recall the problem of existence of the wave operator for (DKG). We define the free-Dirac-and KleinGordon evolution groups as follows:

$$
\begin{gathered}
\mathscr{V}_{D}(t) \equiv I \cos \left(t\langle\nabla\rangle_{M}\right)-(\alpha \cdot \nabla+i M \beta)\langle\nabla\rangle_{M}^{-1} \sin \left(t\langle\nabla\rangle_{M}\right), \\
\mathscr{V}_{K}(t) \equiv\left(\begin{array}{cc}
\cos \left(\langle\nabla\rangle_{m} t\right) & \sin \left(\langle\nabla\rangle_{m} t\right) \\
-\sin \left(\langle\nabla\rangle_{m} t\right) & \cos \left(\langle\nabla\rangle_{m} t\right)
\end{array}\right) .
\end{gathered}
$$

For given final data $\left(\psi^{+},\left(\langle\nabla\rangle_{m} \phi_{1}^{+}, \phi_{2}^{+}\right)\right) \in(\mathbf{X})^{4}$ with some Banach spaces $\mathbf{X}$ defined explicitly later, we put

$$
\begin{aligned}
\psi_{0}(t) & \equiv \mathscr{V}_{D}(t) \psi^{+} \\
\left(\begin{array}{c}
\phi_{0}(t) \\
\langle\nabla\rangle_{m}^{-1} \partial_{t} \phi_{0}(t)
\end{array}\right) & \equiv \mathscr{V}_{K}(t)\left(\begin{array}{c}
\phi_{1}^{+} \\
\langle\nabla\rangle_{m}^{-1} \phi_{2}^{+}
\end{array}\right) .
\end{aligned}
$$

We will look for a unique time local solution of (DKG) which satisfies the final state conditions as follows:

$$
\begin{gathered}
\lim _{t \rightarrow \infty}\left\|\psi(t)-\psi_{0}(t)\right\|_{\widetilde{\mathbf{x}}}=0 \\
\lim _{t \rightarrow \infty}\left\|\left(\begin{array}{c}
\langle\nabla\rangle_{m}^{1 / 2} \phi(t) \\
\langle\nabla\rangle_{m}^{-1 / 2} \partial_{t} \phi(t)
\end{array}\right)-\left(\begin{array}{c}
\langle\nabla\rangle_{m}^{1 / 2} \phi_{0}(t) \\
\langle\nabla\rangle_{m}^{-1 / 2} \partial_{t} \phi_{0}(t)
\end{array}\right)\right\|_{\widetilde{\mathbf{x}}}=0,
\end{gathered}
$$

where $\widetilde{\mathbf{X}}$ is also a suitable Banach space. If there exist $T>0$ and a unique solution $\left(\psi,\langle\nabla\rangle_{m}^{1 / 2} \phi,\langle\nabla\rangle_{m}^{-1 / 2} \partial_{t} \phi\right) \quad \epsilon$ $(\mathbf{C}([T, \infty) ; \widetilde{\mathbf{X}}))^{4}$ for $(\mathrm{DKG})$ satisfying (8)-(9), then the wave operator $\mathscr{W}^{+}$for $(\mathrm{DKG})$ is defined by the mapping as follows:

$$
\begin{aligned}
& \mathscr{W}^{+}:(\mathbf{X})^{2} \times\left(\langle\nabla\rangle^{-1} \mathbf{X} \times \mathbf{X}\right) \\
& \longrightarrow(\widetilde{\mathbf{X}})^{2} \times\left(\langle\nabla\rangle^{-1 / 2} \widetilde{\mathbf{X}} \times\langle\nabla\rangle^{1 / 2} \widetilde{\mathbf{X}}\right), \\
& \left(\psi(t),\left(\phi(t), \partial_{t} \phi(t)\right)\right)=\mathscr{W}^{+}\left(\psi^{+},\left(\phi_{1}^{+}, \phi_{2}^{+}\right)\right), \\
& \text {for } t \in[T, \infty),
\end{aligned}
$$

where $\langle\nabla\rangle^{-s} \mathbf{X} \equiv\left\{\phi ;\left\|\langle\nabla\rangle^{s} \phi\right\|_{\mathbf{X}}<\infty\right\}$.

\section{Several Notations and Main Results}

We introduce several notations to state our main results. For $m, k \in \mathbb{R}$, and $1 \leq p \leq \infty$, we introduce the weighted Sobolev space as follows:

$$
H_{p}^{m, k}=\left\{\phi ;\|\phi\|_{H_{p}^{m, k}} \equiv\left\|\langle x\rangle^{k}\langle\nabla\rangle^{m} \phi\right\|_{L^{p}}<\infty\right\},
$$

where $\langle x\rangle=\left(1+|x|^{2}\right)^{1 / 2},\langle\nabla\rangle=(1-\Delta)^{1 / 2}$. We also write for simplicity $H^{m, k}=H_{2}^{m, k}, H^{m}=H_{2}^{m, 0}$, and $H_{p}^{m}=H_{p}^{m, 0}$, and so we usually omit the index 0 and $p=2$ if it does not cause a confusion.

We now state our main results in this paper. We introduce the function space as follows:

$$
D_{q} \equiv H_{q /(q-1)}^{4-4 / q} \cap H^{5 / 2,1} \cap H_{1}^{2} .
$$

Theorem 1. Let $m, M>0, m \neq 2 M, 4<q \leq \infty$ and $\left(\psi^{+},\left(\langle\nabla\rangle \phi_{1}^{+}, \phi_{2}^{+}\right)\right) \in\left(D_{q}\right)^{4}$. If the norm $\rho \equiv$ $\left\|\left(\psi^{+},\left(\langle\nabla\rangle \phi_{1}^{+}, \phi_{2}^{+}\right)\right)\right\|_{H_{1}^{2}}$ is sufficiently small, then there exist a positive constant $T>0$ and a unique solution

$$
\left(\psi(t),\left(\begin{array}{c}
\langle\nabla\rangle_{m}^{1 / 2} \phi(t) \\
\langle\nabla\rangle_{m}^{-1 / 2} \partial_{t} \phi(t)
\end{array}\right)\right) \in\left(C\left([T, \infty) ; H^{1 / 2}\right)\right)^{4},
$$

for the system (DKG). Moreover, there exists a positive constant $C>0$ such that the following estimate

$$
\begin{aligned}
& \left\|\psi(t)-\psi_{0}(t)\right\|_{H^{1 / 2}}+\left\|\left(\begin{array}{c}
\phi(t) \\
\langle\nabla\rangle_{m}^{-1} \partial_{t} \phi(t)
\end{array}\right)-\left(\begin{array}{c}
\phi_{0}(t) \\
\langle\nabla\rangle_{m}^{-1} \partial_{t} \phi_{0}(t)
\end{array}\right)\right\|_{H^{1}} \\
& \quad \leq C t^{-\mu}
\end{aligned}
$$

is true for all $t \geq T$, where $1 / 2<\mu<1-2 / q$ and $\left(\psi_{0}, \phi_{0}\right)$ is given by (7).

By Theorem 1, we can get existence of the wave operator for (DKG) as follows.

Corollary 2. Let $m, M>0, m \neq 2 M$, and $4 \leq q<\infty$. Then the wave operator $\mathscr{W}^{+}$for $(D K G)$ is well defined from a neighborhood at the origin in the space $\left(D_{q}\right)^{2} \times\left(\langle\nabla\rangle^{-1} D_{q} \times D_{q}\right)$ to the space $\left(H^{1 / 2}\right)^{2} \times\left(H^{1} \times L^{2}\right)$. 
The rest of this paper is organized as follows. In Section 3 , we state some basic estimates for free solutions of the DKG system and we introduce "null forms" and state their properties. In Section 4, we decompose two harmful terms by the algebraic normal form transformation and we find a second approximation for $\psi$ through the decomposition of the KleinGordon operator by the Dirac one. In Section 5, following paper [10], we will also change the transformed DKG system into another form in order to apply the Strichartz type estimates to the Dirac part. In Section 6, we will prove Theorem 1 by an iteration scheme based on paper [13].

\section{Elementary Estimates and Null Forms}

Through the paper, we write $A \simeq B$ if there exist some positive constants $C_{1}, C_{2}>0$ such that $C_{1} B \leq A \leq C_{2} B$, and we also write $A \lesssim B$ if there exists a positive constant $C>0$ such that $A \leq C B$.

We introduce the free evolution groups as follows:

$$
\mathcal{U}_{ \pm, m}(t) \equiv e^{ \pm i t\langle\nabla\rangle_{m}}=\mathscr{F}^{-1} e^{ \pm i t\langle\xi\rangle_{m}} \mathscr{F} .
$$

Then, we have the following decomposition:

$$
\mathscr{V}_{D}(t)=\sum_{ \pm} \mathscr{U}_{ \pm, M}(t) \mathscr{A}_{ \pm}^{D}
$$

where

$$
\mathscr{A}_{ \pm}^{D} \equiv \frac{1}{2}\left(I \pm i\langle\nabla\rangle_{M}^{-1}(\alpha \cdot \nabla+i M \beta)\right)
$$

is 0 th order matrix operator. We note that for any $\mathbb{C}^{2}$-valued function $\psi$, the following equivalency is valid:

$$
\left\|\mathscr{A}_{ \pm}^{D} \psi\right\|_{H_{p}^{m, k}} \simeq\|\psi\|_{H_{p}^{m, k}}
$$

Now, we state $L^{p}-L^{q}$ time decay estimates through the free evolution groups $\mathscr{U}_{ \pm, m}(t)$ obtained in paper [14].

Lemma 3. Let $m \neq 0$ and $2 \leq p \leq \infty$. Then the estimate

$$
\left\|\mathcal{U}_{ \pm, m}(t) \phi\right\|_{L^{p}} \lesssim t^{2 / p-1}\|\phi\|_{H_{q}^{2(1-2 / p)}}
$$

is true for any $t>0$, where $q$ is a conjugate exponent of $p$ : $1 / p+1 / q=1$.

By the lemma, we can easily get $L^{p}-L^{q}$ time decay estimates to free solutions for the DKG system.

Corollary 4. Under the same assumption of Lemma 3 and $M>0$, the following estimates

$$
\begin{gathered}
\left\|\mathscr{V}_{D}(t) \psi^{+}\right\|_{L^{p}} \lesssim t^{2 / p-1}\left\|\psi^{+}\right\|_{H_{q}^{2(1-2 / p)}} \\
\left\|\mathscr{V}_{K}(t)\left(\begin{array}{c}
\phi_{1}^{+} \\
\langle\nabla\rangle_{m}^{-1} \phi_{2}^{+}
\end{array}\right)\right\|_{L^{p}} \lesssim t^{2 / p-1}\left\|\left(\begin{array}{c}
\phi_{1}^{+} \\
\langle\nabla\rangle_{m}^{-1} \phi_{2}^{+}
\end{array}\right)\right\|_{H_{q}^{2(1-2 / p)}}
\end{gathered}
$$

are valid for any $t>0$, where $q$ is a conjugate exponent of $p$ : $1 / p+1 / q=1$.
Remark 5. Let $\kappa \in \mathbb{R}, M, m \neq 0$, and $2 \leq p<\infty$. Then the following estimates

$$
\begin{gathered}
\left\|\mathscr{V}_{D}(t) \psi^{+}\right\|_{H_{p}^{\kappa}} \lesssim t^{2 / p-1}\left\|\psi^{+}\right\|_{H^{\kappa+2-4 / p, 1}}, \\
\left\|\mathscr{V}_{K}(t)\left(\begin{array}{c}
\phi_{1}^{+} \\
\langle\nabla\rangle_{m}^{-1} \phi_{2}^{+}
\end{array}\right)\right\|_{H_{p}^{\kappa}} \lesssim t^{2 / p-1}\left\|\left(\begin{array}{c}
\phi_{1}^{+} \\
\langle\nabla\rangle_{m}^{-1} \phi_{2}^{+}
\end{array}\right)\right\|_{H^{\kappa+2-4 / p, 1}}
\end{gathered}
$$

hold for any $t>0$.

Next, we introduce the Strichartz estimates, which enable us to treat the problem in lower order Sobolev spaces. Denote the space-time norm

$$
\|\phi\|_{L_{t}^{r}\left(I ; L_{x}^{q}\right)} \equiv\|\| \phi(t)\left\|_{L_{x}^{q}}\right\|_{L_{t}^{r}(I)},
$$

where $I$ is a bounded or unbounded time interval. We define the integral operator as follows:

$$
\mathscr{G}_{ \pm, m}[g](t) \equiv \int_{T}^{t} \mathscr{U}_{ \pm, m}(t-\tau)\langle\nabla\rangle_{m}^{-1} g(\tau) d \tau
$$

for any $T \in \bar{I}$, where $m>0$. By the duality argument of [15] along with Lemma 3 , we have the following (see also $[10,13]$ ).

Lemma 6. Let $2 \leq q<\infty$ and $2 / r=1-(2 / q)$. Then for any time interval $I$, the following estimates are true:

$$
\begin{aligned}
\left\|\mathscr{G}_{ \pm, m}[g]\right\|_{L_{t}^{r}\left(I ; L_{x}^{q}\right)} & \leqslant\|g\|_{L_{t}^{r^{\prime}}\left(I ; H_{q^{\prime}}^{2 \gamma-1}\right)}, \\
\left\|\mathscr{G}_{ \pm, m}[g]\right\|_{L_{t}^{\infty}\left(I ; L_{x}^{2}\right)} & \leqslant\|g\|_{L_{t}^{r^{\prime}\left(I ; H_{q^{\prime}}^{\gamma-1}\right)}}, \\
\left\|\mathscr{G}_{ \pm, m}[g]\right\|_{L_{t}^{r}\left(I ; L_{x}^{q}\right)} & \leqslant\|g\|_{L_{t}^{1}\left(I ; H^{\gamma-1}\right)},
\end{aligned}
$$

where $r^{\prime}=r /(r-1), q^{\prime}=q /(q-1)$ and $\gamma=1-(2 / q)$.

Next, we introduce the Leibniz rule for fractional derivatives.

Lemma 7. Let $\kappa>0,1<p, q_{1}, q_{2}<\infty, 1<r_{1}, r_{2} \leq \infty$, and $1 / p=1 / q_{1}+1 / r_{1}=1 / q_{2}+1 / r_{2}$. Then the following estimate holds:

$$
\|u v\|_{H_{p}^{\kappa}} \lesssim\|u\|_{H_{q_{1}}^{\kappa}}\|v\|_{L^{r_{1}}}+\|v\|_{H_{q_{2}}^{\kappa}}\|u\|_{L^{r_{2}}} .
$$

For the proof of (25) see, for example, [16].

We introduce the operator $\mathscr{Z}=\left(\mathscr{Z}_{1}, \mathscr{Z}_{2}\right)$, where $\mathscr{Z}_{k} \equiv$ $x_{k} \partial_{t}+t \partial_{k}$ for $k=1,2$. Let $\mathscr{Z}^{\alpha}=\mathscr{Z}_{1}^{\alpha_{1}} \mathscr{Z}_{2}^{\alpha_{2}}$ for a multiindex $\alpha=\left(\alpha_{1}, \alpha_{2}\right) \in(\mathbb{N} \cup\{0\})^{2}$. We can see the commutation relations (see $[6,17])$ as follows:

$$
\begin{gathered}
{\left[\mathscr{D}_{+}, \mathscr{Z}_{k}-\left(\frac{1}{2}\right) \alpha_{k}\right]=\alpha_{k} \mathscr{D}_{+},} \\
{\left[\partial_{t}^{2}-\Delta+m^{2}, \mathscr{Z}_{k}\right]=0,}
\end{gathered}
$$

for $k=1,2$, where $[A, B] \equiv A B-B A$.

We introduce the quadratic null forms as follows:

$$
\begin{gathered}
\mathcal{Q}_{0}(f, g) \equiv\left(\partial_{t} f\right)\left(\partial_{t} g\right)-(\nabla f) \cdot(\nabla g), \\
\mathcal{Q}_{j, k}(f, g) \equiv\left(\partial_{j} f\right)\left(\partial_{k} g\right)-\left(\partial_{k} f\right)\left(\partial_{j} g\right),
\end{gathered}
$$


for $0 \leq j<k \leq 2$, where $\partial \equiv\left(\partial_{0}, \nabla\right) \equiv\left(i \partial_{t}, \partial_{1}, \partial_{2}\right)$. In particular, $\mathbb{Q}_{j, k}$ is called a strong null form and has an additional time decay property through the operator $\mathscr{Z}_{k}$, obtained in [18] (see also $[8,13,19]$, etc.).

Lemma 8. Let $j, k=1,2$. Then, for any smooth function $f, g$, the identities

$$
\begin{aligned}
\mathcal{Q}_{0, j}(f, g)= & t^{-1}\left(\partial_{0} f\right)\left(\mathscr{Z}_{j} g\right)-t^{-1}\left(\mathscr{Z}_{j} f\right)\left(\partial_{0} g\right), \\
\mathcal{Q}_{j, k}(f, g)= & t^{-2}\left(\mathscr{Z}_{j} g\right)\left(\mathscr{Z}_{k} f\right) \\
& -t^{-2}\left(\mathscr{Z}_{j} f\right)\left(\mathscr{Z}_{k} g\right)+t^{-1}\left(\partial_{j} f\right)\left(\mathscr{Z}_{k} g\right) \\
& -t^{-1}\left(\partial_{j} g\right)\left(\mathscr{Z}_{k} f\right)+t^{-1}\left(\mathscr{X}_{j} f\right)\left(\partial_{k} g\right) \\
& -t^{-1}\left(\mathscr{Z}_{j} g\right)\left(\partial_{k} f\right)
\end{aligned}
$$

are valid for any $t \in \mathbb{R} \backslash\{0\}$.

\section{Decomposition of Critical Terms}

We study a structure of some harmful terms of (DKG). By the difference of (DKG) and the free DKG system, it follows that

$$
\begin{aligned}
\mathscr{D}_{+}\left(\psi-\psi_{0}\right)= & \left(\phi-\phi_{0}\right) \beta \psi \\
& +\phi_{0} \beta\left(\psi-\psi_{0}\right)+\phi_{0} \beta \psi_{0}, \\
\left(\square+m^{2}\right)\left(\phi-\phi_{0}\right)= & \left(\psi-\psi_{0}\right)^{*} \beta \psi \\
& +\psi_{0}^{*} \beta\left(\psi-\psi_{0}\right)+\psi_{0}^{*} \beta \psi_{0},
\end{aligned}
$$

where $\square=\partial_{t}^{2}-\Delta$. The last two terms $\phi_{0} \beta \psi_{0}$ and $\psi_{0}^{*} \beta \psi_{0}$ are critical, both of which have the worst time decay property. Especially, since

$$
\phi_{0} \beta \psi_{0}, \psi_{0}^{*} \beta \psi_{0}=O\left(t^{-1}\right) \quad \text { in } L^{2} \text { as } t \longrightarrow+\infty
$$

(see Corollary 4), the $L^{2}$-norm of these terms is not integrable with respect to time $t$ over $[1, \infty)$. Therefore, it can not be expected that usual perturbation technique is applicable to (29). To overcome this lack of time decay property, we will decompose them into an image of a Klein-Gordon operator and a remainder term following paper [8], based on papers [19-21].

Let $\left(v_{1}, v_{2}\right)$ be a solution for the following homogeneous KG system with masses $M_{1}, M_{2}>0$,

$$
\left(\square+M_{j}^{2}\right) v_{j}=0, \quad(t, x) \in \mathbb{R} \times \mathbb{R}^{2}, \quad \text { for } j=1,2 .
$$

By the masses $M_{1}, M_{2}$, we introduce the symmetric matrix as follows:

$$
\mathscr{M}=\mathscr{M}\left(M_{1}, M_{2}\right)=\left(\begin{array}{cc}
M_{1}^{2}+M_{2}^{2} & 2 M_{1} M_{2} \\
2 M_{1} M_{2} & M_{1}^{2}+M_{2}^{2}
\end{array}\right) .
$$

We have the following.

Lemma 9 (see [8]). Let $\widetilde{m}>0$ with $\operatorname{det}\left(\widetilde{m}^{2} I-\mathscr{M}\right) \neq 0$. Then the quadratic term $v_{1} v_{2}$ can be decomposed as

$$
v_{1} v_{2}=\frac{1}{\operatorname{det}\left(\widetilde{m}^{2} I-\mathscr{M}\right)}\left\{\left(\square+\widetilde{m}^{2}\right) f-4 \mathscr{R}\right\},
$$

where

$$
\begin{aligned}
& f=f\left(v_{1}, v_{2}\right) \equiv\left(-M_{1}^{2}-M_{2}^{2}+\widetilde{m}^{2}\right) v_{1} v_{2}-2 Q_{0}\left(v_{1}, v_{2}\right), \\
& \mathscr{R}= \mathscr{R}\left(v_{1}, v_{2}\right) \equiv \sum_{m=1}^{2} Q_{0, m}\left(\partial_{t} v_{1}, \partial_{m} v_{2}\right) \\
&+\sum_{m=1}^{2} Q_{0, m}\left(\partial_{t} v_{2}, \partial_{m} v_{1}\right)-Q_{1,2}\left(\partial_{1} v_{1}, \partial_{2} v_{2}\right) \\
&-Q_{2,1}\left(\partial_{2} v_{1}, \partial_{1} v_{2}\right) .
\end{aligned}
$$

Under the nonresonance mass condition $m, M>0$, and $m \neq 2 M$, we can apply Lemma 9 to the critical terms $\phi_{0} \beta \psi_{0}$ and $\psi_{0}^{*} \beta \psi_{0}$. Before doing so, we prepare for several notations. We put

$$
\widetilde{M} \equiv \frac{1}{m^{2}(2 M+m)(m-2 M)}
$$

which is well defined if $m, M>0$ and $m \neq 2 M$. For a realvalued function $\phi$ and a $\mathbb{C}^{2}$-valued function $\psi=\left(\psi_{1}, \psi_{2}\right)^{t}$, we define $\mathbb{C}^{2}$-valued functions of bilinear form:

$$
\begin{gathered}
f_{D}=f_{D}(\phi, \psi) \equiv\left(f\left(\phi, \psi_{1}\right), f\left(\phi, \psi_{2}\right)\right)^{t}, \\
\mathscr{R}_{D}=\mathscr{R}_{D}(\phi, \psi) \equiv\left(\mathscr{R}\left(\phi, \psi_{1}\right), \mathscr{R}\left(\phi, \psi_{2}\right)\right)^{t}, \\
{Q_{0}^{D}}^{D}=\mathscr{Q}_{0}^{D}(\phi, \psi) \equiv\left(Q_{0}\left(\phi, \psi_{1}\right), \mathscr{Q}_{0}\left(\phi, \psi_{2}\right)\right)^{t},
\end{gathered}
$$

Moreover, for $\mathbf{C}^{2}$-valued functions $\varphi=\left(\varphi_{1}, \varphi_{2}\right)^{t}, \psi=$ $\left(\psi_{1}, \psi_{2}\right)^{t}$, we put the following bilinear forms:

$$
\begin{gathered}
f_{K}=f_{K}\left(\varphi^{t}, \psi\right) \equiv \sum_{j=1}^{2} f\left(\varphi_{j}, \psi_{j}\right), \\
\mathscr{R}_{K}=\mathscr{R}_{K}\left(\varphi^{t}, \psi\right) \equiv \sum_{j=1}^{2} \mathscr{R}\left(\varphi_{j}, \psi_{j}\right), \\
{Q_{0}^{K}}^{K}=Q_{0}^{K}\left(\varphi^{t}, \psi\right) \equiv \sum_{j=1}^{2} Q_{0}\left(\varphi_{j}, \psi_{j}\right) .
\end{gathered}
$$

We have the following.

Corollary 10. Let $m, M>0, m \neq 2 M$, and $\left(\psi_{0}, \phi_{0}\right)$ be a free solution for the Dirac-Klein-Gordon equations. Then the quadratic terms $\phi_{0} \beta \psi_{0}, \psi_{0}^{*} \beta \psi_{0}$ can be expressed as

$$
\begin{aligned}
& \phi_{0} \beta \psi_{0}=\widetilde{\mathscr{M}}\left\{\left(\square+M^{2}\right) f_{D}\left(\phi_{0}, \beta \psi_{0}\right)-4 \mathscr{R}_{D}\left(\phi_{0}, \beta \psi_{0}\right)\right\}, \\
& \psi_{0}^{*} \beta \psi_{0}=\widetilde{M}\left\{\left(\square+m^{2}\right) f_{K}\left(\psi_{0}^{*}, \beta \psi_{0}\right)-4 \mathscr{R}_{K}\left(\psi_{0}^{*}, \beta \psi_{0}\right)\right\} .
\end{aligned}
$$


Proof. We consider the Dirac part of (38). Multiplying by $\mathscr{D}_{-}$ both hand sides of $\mathscr{D}_{+} \psi_{0}=0$, we get

$$
\mathscr{D}_{-} \mathscr{D}_{+} \psi_{0}=\left(\square+M^{2}\right) \psi_{0}=0 \text {, }
$$

which implies that $\psi_{0}=\left(\psi_{0,1}, \psi_{0,2}\right)^{t}$ is also a solution of the free $\mathrm{KG}$ equation. Note that by the condition $m, M>0$ and $m \neq 2 M$, we can apply Lemma 9 with $\widetilde{m}=M, v_{1}=\phi_{0}$, and $v_{2}=\psi_{0, k}$ to get, for $k=1,2$,

$$
\phi_{0} \psi_{0, k}=\widetilde{\mathscr{M}}\left\{\left(\square+M^{2}\right) f\left(\phi_{0}, \psi_{0, k}\right)-4 \mathscr{R}\left(\phi_{0}, \psi_{0, k}\right)\right\} .
$$

Thus, by a simple calculation, we obtain (38). Next, note that from equality (39), we see that $\overline{\psi_{0}}$ satisfies the free KG equation. Thus in the same manner as the proof of the Dirac part, we can prove the KG part, which completes the proof of the corollary.

Next, we will change the DKG equations into another form without critical nonlinearities. We introduce a new unknown function $(\Psi, \Phi)$ as follows:

$$
\begin{aligned}
& \Psi \equiv \psi-\psi_{0}-\tilde{f}_{D} \equiv \widetilde{\psi}-\tilde{f}_{D}, \\
& \Phi \equiv \phi-\phi_{0}-\widetilde{f}_{K} \equiv \widetilde{\phi}-\widetilde{f}_{K},
\end{aligned}
$$

where $\left(\psi_{0}, \phi_{0}\right)$ is defined by $(7)$ and

$$
\begin{gathered}
\tilde{f}_{D}=\widetilde{f}_{D}\left(\phi_{0}, \psi_{0}\right) \equiv \widetilde{\mathscr{M}} \mathscr{D}_{-} f_{D}\left(\phi_{0}, \beta \psi_{0}\right) \\
=\widetilde{\mathscr{M}}\left(f_{D}\left(\mathscr{D}_{-} \phi_{0}, \beta \psi_{0}\right)-i M f_{D}\left(\phi_{0}, \psi_{0}\right)\right), \\
\tilde{f}_{K}=\tilde{f}_{K}\left(\psi_{0}\right) \equiv \widetilde{\mathscr{M}} f_{K}\left(\psi_{0}^{*}, \beta \psi_{0}\right)
\end{gathered}
$$

are the second approximate solution to $(\psi, \phi)$, where we have used the identities $\alpha_{j} \beta+\beta \alpha_{j}=O, \beta^{2}=I$ and $\mathscr{D}_{+} \psi_{0}=0$ to obtain the third equality in (42).

Here, we remember that by the anticommutation relations (1) of the Dirac matrices, we can decompose the KG operator as follows:

$$
\square+M^{2}=\mathscr{D}_{+} \mathscr{D}_{-} .
$$

By combining Corollary 10 and this decomposition, we can rewrite $(\mathrm{DKG})$ as follows.

Lemma 11. Let $m, M>0$ and $m \neq 2 M$. Then $(\psi, \phi)$ satisfies $(D K G)$ if and only if the new variable $(\Psi, \Phi)$ defined by (41) is a solution of

$$
\begin{gathered}
\mathscr{D}_{+} \Psi=F, \\
\left(\square+m^{2}\right) \Phi=G, \quad(t, x) \in \mathbb{R} \times \mathbb{R}^{2},
\end{gathered}
$$

where

$$
\begin{aligned}
F & =F(\widetilde{\phi}, \widetilde{\psi}) \\
& \equiv \widetilde{\phi} \beta \widetilde{\psi}+\widetilde{\phi} \beta \psi_{0}+\phi_{0} \beta \widetilde{\psi}-4 \widetilde{\mathscr{M}} \mathscr{R}_{D}\left(\phi_{0}, \beta \psi_{0}\right), \\
G & =G(\widetilde{\psi}) \\
& \equiv \widetilde{\psi}^{*} \beta \widetilde{\psi}+\widetilde{\psi}^{*} \beta \psi_{0}+\psi_{0}^{*} \beta \widetilde{\psi}-4 \widetilde{\mathscr{M}} \mathscr{R}_{K}\left(\psi_{0}^{*}, \beta \psi_{0}\right),
\end{aligned}
$$

and $\widetilde{\mathscr{M}}, \mathscr{R}_{D}$ and $\mathscr{R}_{K}$ are defined by (35), (36), and (37), respectively.
This lemma enables us to treat the Dirac-Klein-Gordon equations (DKG) as well as the reduced KG system (4) in two space dimensions.

Proof. From (29), we see that $(\psi, \phi)$ is a solution of (DKG) if and only if the new variable $(\widetilde{\psi}, \widetilde{\phi})$ satisfies the following DKG equations:

$$
\begin{gathered}
\mathscr{D}_{+} \tilde{\psi}=\tilde{\phi} \beta \tilde{\psi}+\widetilde{\phi} \beta \psi_{0}+\phi_{0} \beta \tilde{\psi}+\phi_{0} \beta \psi_{0}, \\
\left(\square+m^{2}\right) \tilde{\phi}=\widetilde{\psi}^{*} \beta \widetilde{\psi}+\widetilde{\psi}^{*} \beta \psi_{0}+\psi_{0}^{*} \beta \widetilde{\psi}+\psi_{0}^{*} \beta \psi_{0} .
\end{gathered}
$$

We consider the Dirac part of (47) only, since it is easier to handle the KG part. Note that by the assumption $m, M>0$ and $m \neq 2 M$, we can apply Corollary 10 to $\phi_{0} \beta \psi_{0}$. Thus, we have

$$
\phi_{0} \beta \psi_{0}=\widetilde{\mathscr{M}}\left\{\left(\square+M^{2}\right) f_{D}\left(\phi_{0}, \beta \psi_{0}\right)-4 \mathscr{R}_{D}\left(\phi_{0}, \beta \psi_{0}\right)\right\} .
$$

Moreover, by the decomposition (44), we can transform the first term of the right hand side of (48) as follows:

$$
\begin{aligned}
\lambda \widetilde{\mathscr{M}} & \left(\square+M^{2}\right) f_{D}\left(\phi_{0}, \beta \psi_{0}\right) \\
& =\widetilde{\mathscr{M}} \mathscr{D}_{+} \mathscr{D}_{-} f_{D}\left(\phi_{0}, \beta \psi_{0}\right)=\mathscr{D}_{+} \widetilde{f}_{D},
\end{aligned}
$$

where we have used the definition of $\tilde{f}_{D}$ given by (42). Inserting (48) and (49) into the Dirac part of (47), we obtain the Dirac part of (45), which completes the proof of the lemma.

Remark 12. The null structure of (DKG) was characterized in [12] by using Fourier space. On the other hand, we note that in the above argument, Fourier space does not appear at all.

\section{Reduction to Some First Order System}

To construct a solution for the final value problem of the DKG system, we will use the Strichartz type estimates (Lemma 6). However, it seems difficult to apply these estimates to the Dirac part for (45) due to a derivative loss difficulty. To gain first order differentiability properties of nonlinear term, we use the matrix operators

$$
\begin{gathered}
\mathscr{B}_{ \pm}^{D} \equiv \frac{1}{2} I\left(1 \mp i\langle\nabla\rangle_{M}^{-1} \partial_{t}\right)=\mp \frac{i}{2}\langle\nabla\rangle_{M}^{-1} \mathscr{L}_{\mp}^{D}, \\
\mathscr{L}_{ \pm}^{D} \equiv\left(\partial_{t} \mp i\langle\nabla\rangle_{m}\right) I,
\end{gathered}
$$

though we do not necessarily need the operator $\mathscr{B}$ in dealing with the initial value problem for the DKG system (see [11]). We will construct the desired solution $(\psi, \phi)$ for the DKG system by the iteration scheme. Let $\left\{\left(\psi^{l}, \phi^{l}\right)\right\}_{l \geq 0}$ be a sequence such that

$$
\begin{gathered}
\mathscr{D}_{+} \psi^{l+1}=\phi^{l} \beta \psi^{l}, \\
\left(\square+m^{2}\right) \phi^{l+1}=\left(\psi^{l}\right)^{*} \beta \psi^{l}, \quad l \geq 0, \\
\left(\psi^{0}, \phi^{0}\right)=\left(\psi_{0}, \phi_{0}\right),
\end{gathered}
$$


under the final conditions

$$
\begin{gathered}
\lim _{t \rightarrow \infty}\left\|\psi^{l}(t)-\psi_{0}(t)\right\|_{H^{1 / 2}}=0 \\
\lim _{t \rightarrow \infty}\left\|\left(\begin{array}{c}
\phi^{l}(t) \\
\langle\nabla\rangle_{m}^{-1} \partial_{t} \phi^{l}(t)
\end{array}\right)-\left(\begin{array}{c}
\phi_{0}(t) \\
\langle\nabla\rangle_{m}^{-1} \partial_{t} \phi_{0}(t)
\end{array}\right)\right\|_{H^{1}}=0,
\end{gathered}
$$

for $l \geq 0$, where $\left(\psi_{0}, \phi_{0}\right)$ is given by (7). It suffices to prove that the sequence $\left\{\psi^{l},\left(\langle\nabla\rangle_{m}^{1 / 2} \phi^{l},\langle\nabla\rangle_{m}^{-1 / 2} \partial_{t} \phi^{l}\right)\right\}_{l \geq 0}$ is a Cauchy one in the Banach space $\left(C\left([T, \infty) ; H^{1 / 2}\right)\right)^{4}$ for some $T>0$.

As the previous section, we introduce the new sequence $\left\{\left(\Psi^{l}, \Phi^{l}\right)\right\}$ as follows:

$$
\begin{aligned}
& \Psi^{l} \equiv \psi^{l}-\psi_{0}-\tilde{f}_{D} \equiv \widetilde{\psi}^{l}-\tilde{f}_{D}, \\
& \Phi^{l} \equiv \phi^{l}-\phi_{0}-\tilde{f}_{K} \equiv \widetilde{\phi}^{l}-\tilde{f}_{K} .
\end{aligned}
$$

By Lemma 11, the sequence $\left\{\left(\psi^{l}, \phi^{l}\right)\right\}$ is a solution of (51) if and only if the new one $\left\{\left(\Psi^{l}, \Phi^{l}\right)\right\}$ satisfies the transformed DKG equations as follows:

$$
\begin{aligned}
& \mathscr{D}_{+} \Psi^{l+1}=F^{l}, \\
& \left.\square+m^{2}\right) \Phi^{l+1}=G^{l}, \quad l \geq 1, \\
& \left(\Psi^{0}, \Phi^{0}\right)=-\left(\widetilde{f}_{D}, \widetilde{f}_{K}\right),
\end{aligned}
$$

where

$$
F^{l} \equiv F\left(\widetilde{\phi}^{l}, \widetilde{\psi}^{l}\right), \quad G^{l} \equiv G\left(\widetilde{\psi}^{l}\right),
$$

for $l \geq 0\left(\widetilde{f}_{D}, \widetilde{f}_{K}\right.$, and $F$ and $G$ are defined by (42)-(43) and (46), resp.).

By the decomposition of the Klein-Gordon operator by the Dirac operator, we have

$$
\mathscr{L}_{ \pm}^{D} \mathscr{B}_{ \pm}^{D}=\mp \frac{i}{2}\langle\nabla\rangle_{M}^{-1} I\left(\partial_{t}^{2}+\langle\nabla\rangle_{M}^{2}\right)=\mp \frac{i}{2}\langle\nabla\rangle_{M}^{-1} \mathscr{D}_{-} \mathscr{D}_{+} .
$$

Thus, from the Dirac part for (55), we can deduce the following:

$$
\mathscr{L}_{ \pm}^{D} \mathscr{B}_{ \pm}^{D} \Psi^{l+1}=\mp \frac{i}{2}\langle\nabla\rangle_{M}^{-1} \mathscr{D}_{-} \mathscr{D}_{+} \Psi^{l+1}=\langle\nabla\rangle_{M}^{-1} F_{ \pm}^{l},
$$

for $l \geq 0$, where $F_{ \pm}^{l} \equiv \mp(i / 2) \mathscr{D}_{-} F^{l}$. Therefore, from Dirac part of (55), we have

$$
\begin{gathered}
\mathscr{L}_{ \pm}^{D} \mathscr{B}_{ \pm}^{D} \Psi^{l+1}=\langle\nabla\rangle_{M}^{-1} F_{ \pm}^{l}, \quad l \geq 0, \\
\mathscr{B}_{ \pm}^{D} \Psi^{0}=-\mathscr{B}_{ \pm}^{D} \widetilde{f}_{D} .
\end{gathered}
$$

Remark 13. By properties (1) of the Dirac matrices, we can transform $F_{ \pm}^{l}$ into another form without any derivatives of $\widetilde{\psi}$ or the free solution $\psi_{0}$ (see (78)-(79), precisely). This fact enables us to use the Strichartz estimates for (60).

Next we will also transform the KG part of (55) as in $[10,13]$. We also use the operator (1-component version of the Dirac part) as follows:

$$
\mathscr{B}_{ \pm}^{K} \equiv \frac{1}{2}\left(1 \mp i\langle\nabla\rangle_{m}^{-1} \partial_{t}\right), \quad \mathscr{L}_{ \pm}^{K} \equiv \partial_{t} \mp i\langle\nabla\rangle_{m} .
$$

We can see that the sequence $\left\{\Phi^{l}\right\}$ is a solution of the KG part for (55) if and only if the sequence $\left\{\mathscr{B}_{ \pm}^{K} \Phi^{l}\right\}$ satisfies

$$
\begin{gathered}
\mathscr{L}_{ \pm}^{K} \mathscr{B}_{ \pm}^{K} \Phi^{l+1}=\langle\nabla\rangle_{m}^{-1} G_{ \pm}^{l}, \quad \text { for } l \geq 0, \\
\mathscr{B}_{ \pm}^{K} \Phi^{0}=-\mathscr{B}_{ \pm}^{K} \tilde{f}_{K},
\end{gathered}
$$

where $G_{ \pm}^{l} \equiv G_{ \pm}^{l}\left(\widetilde{\psi}^{l}\right) \equiv \mp(i / 2) G^{l}$.

Therefore, by (60) and (62), we get

$$
\begin{aligned}
& \mathscr{L}_{ \pm}^{D} \mathscr{B}_{ \pm}^{D} \Psi^{l+1}=\langle\nabla\rangle_{M}^{-1} F_{ \pm}^{l}, \quad \text { for } l \geq 0, \\
& \mathscr{L}_{ \pm}^{K} \mathscr{B}_{ \pm}^{K} \Phi^{l+1}=\langle\nabla\rangle_{m}^{-1} G_{ \pm}^{l}, \quad \\
& \left(\mathscr{B}_{ \pm}^{D} \Psi^{0}, \mathscr{B}_{ \pm}^{K} \Phi^{0}\right)=-\left(\mathscr{B}_{ \pm}^{D} \widetilde{f}_{D}, \mathscr{B}_{ \pm}^{K} \tilde{f}_{K}\right) .
\end{aligned}
$$

Remark 14. The identity $\sum_{ \pm} \mathscr{B}_{ \pm}^{*}=I$ holds, which enables us to reconstruct a solution $(\Psi, \Phi)$ for $(45)$ from $\left(\mathscr{B}_{ \pm}^{D} \Psi, \mathscr{B}_{ \pm}^{K} \Phi\right)$.

Inserting the identities

$$
\widetilde{\psi}^{l}=\sum_{ \pm} \mathscr{B}_{ \pm}^{D} \Psi^{l}+\tilde{f}_{D}, \quad \widetilde{\phi}^{l}=\sum_{ \pm} \mathscr{B}_{ \pm}^{K} \Phi^{l}+\widetilde{f}_{K},
$$

into the nonlinearities $F_{ \pm}^{l}, G_{ \pm}^{l}$, we can express (63) by the new variable $\left(\mathscr{B}_{+}^{D} \Psi^{l}, \mathscr{B}_{+}^{K} \Phi^{l}\right)$ only without $\left(\widetilde{\phi}^{l}, \widetilde{\psi}^{l}\right)$.

At the end of this section, we will lead the integral equations associated with (63). We introduce a new unknown function sequence $\left\{v^{l}\right\}$ whose components are defined by

$$
v^{l} \equiv\left(\mathscr{B}_{+}^{D} \Psi^{l}, \mathscr{B}_{-}^{D} \Psi^{l},\langle\nabla\rangle_{m}^{1 / 2} \mathscr{B}_{+}^{K} \Phi^{l},\langle\nabla\rangle_{m}^{1 / 2} \mathscr{B}_{-}^{K} \Phi^{l}\right)^{t},
$$

a nonlinear term

$$
\mathcal{N}=\mathcal{N}\left(v^{l}\right) \equiv\left(\langle\nabla\rangle_{M}^{-1} F_{+}^{l},\langle\nabla\rangle_{M}^{-1} F_{-}^{l},\langle\nabla\rangle_{m}^{-1 / 2} G_{+}^{l},\langle\nabla\rangle_{m}^{-1 / 2} G_{-}^{l}\right)^{t}
$$

for $l \geq 0$, and a matrix-operator $\mathscr{L} \equiv \operatorname{diag}\left(\mathscr{L}_{+}^{D}\right.$, $\left.\mathscr{L}_{-}^{D}, \mathscr{L}_{+}^{K}, \mathscr{L}_{-}^{K}\right)$. Then by using these notations, (63) can be simplified as

$$
\mathscr{L} v^{l+1}=\mathscr{N}\left(v^{l}\right) \quad \text { for } l \geq 0 .
$$

To lead the integral equations for (68), we need to study the asymptotic behavior of the new variable $v^{l}$. We can obtain the following.

Lemma 15. Let $\left(\psi^{+},\left(\langle\nabla\rangle \phi_{1}^{+}, \phi_{2}^{+}\right)\right) \in\left(H^{5 / 2,1}\right)^{4}$. The function $\left(\psi^{l}, \phi^{l}\right)$ defined by (51) satisfies (52)-(53) for any $l \geq 0$ if and only if the new function $v^{l}$ satisfies (68) and

$$
\lim _{t \rightarrow \infty}\left\|v^{l}\right\|_{H^{1 / 2}}=0, \quad \text { for } l \geq 0 .
$$

The proof of the lemma will be given in Appendix.

We introduce a matrix evolution operator as follows:

$$
\mathcal{U}(t) \equiv \operatorname{diag}\left(\mathscr{U}_{+, M}(t), \mathcal{U}_{-, M}(t), \mathscr{U}_{+, m}(t), \mathscr{U}_{-, m}(t)\right) .
$$

From Lemma 15, we can lead the integral equations associated with (68) as follows:

$$
v^{l+1}(t)=-\int_{t}^{\infty} \mathcal{U}(t-s) \mathcal{N}\left(v^{l}\right) d s
$$




\section{Proof of Theorem 1}

In this section, we give a proof of Theorem 1 . Note that the identities

$$
\begin{aligned}
& \partial_{t} \Psi^{l}=i\langle\nabla\rangle_{M}\left(v_{1}^{l}-v_{2}^{l}\right), \\
& \partial_{t} \Phi^{l}=i\langle\nabla\rangle_{m}^{1 / 2}\left(v_{3}^{l}-v_{4}^{l}\right)
\end{aligned}
$$

hold; the nonlinearity $\mathcal{N}\left(v^{l}\right)$ can be expressed in terms of the space derivatives of $v^{l}$ (so excluding the time derivatives).

For $T>1$, where $T$ is sufficiently large, we introduce the following function space:

$$
\mathbf{X}_{T}=\left\{v \in\left(C\left([T, \infty) ; H^{1 / 2}\right)\right)^{6} ;\|v\|_{\mathbf{X}_{T}}<\infty\right\}
$$

with the norm

$$
\|v\|_{\mathbf{X}_{T}} \equiv \sup _{t \in[T, \infty)} t^{\mu}\left(\|v\|_{L_{t}^{4}\left(I ; L^{4}\right)}+\|v\|_{L_{t}^{\infty}\left(I ; H^{1 / 2}\right)}\right)
$$

where $1 / 2<\mu<1-2 / q, 4<q \leq \infty$, and $I=[t, \infty)$. We define

$$
A \equiv C\left\|\left(\psi^{+},\left(\langle\nabla\rangle \phi_{1}^{+}, \phi_{2}^{+}\right)\right)\right\|_{H_{q /(q-1)}^{4-4 / q} \cap H^{5 / 2,1}}
$$

In order to obtain the theorem, we will show that the sequence $\left\{v^{l}\right\}$ is a Cauchy one in a closed ball $\mathbf{X}_{T, A}$ for appropriate $T$ and $\rho$, where $\mathbf{X}_{T, A} \equiv\left\{v \in \mathbf{X}_{T} ;\|v\|_{\mathbf{X}_{T}} \leq A\right\}$.

Hereafter, we will use the notation $L_{t}^{r} X=L_{t}^{r}(I ; X)$, $\mathscr{D}=$ $\mathscr{D}_{-}$and

$$
\mathscr{B} \Psi=\mathscr{B}_{ \pm}^{D} \Psi, \quad \mathscr{B} \Phi=\mathscr{B}_{ \pm}^{K} \Phi,
$$

for simplicity if it does not cause a confusion.

Proof. We will prove that $v^{l} \in \mathbf{X}_{T, A}$ for any $l \geq 0$ by induction. In the case of $l=0$, it is easy to see that $v^{0} \in \mathbf{X}_{T, A}$ for some $T$ and $\rho$. We omit the details. For $l \geq 1$, we assume that $v^{k} \in \mathbf{X}_{T, A}$ for $0 \leq k \leq l$. We will show that $v^{l+1} \in \mathbf{X}_{T, A}$ for some $T$ and $\rho$.

First, by the identities $\mathscr{D}_{+} \psi_{0}=0$ and $\mathscr{D}_{+} \psi^{l}=\lambda \phi^{l-1} \beta \psi^{l-1}$ for $l \geq 1$, we get, for $l \geq 1$,

$$
\begin{aligned}
& \mathscr{D}_{-}\left(\tilde{\phi}^{l} \beta \widetilde{\psi}^{l}\right)=\left(\mathscr{D}_{-} \tilde{\phi}^{l}\right) \beta \tilde{\psi}^{l} \\
&-i M \tilde{\phi}^{l} I \tilde{\psi}^{l}+\lambda \tilde{\phi}^{l} \tilde{\phi}^{l-1} I \tilde{\psi}^{l-1} \\
&+\lambda \widetilde{\phi}^{l} \tilde{\phi}^{l-1} I \psi_{0}+\lambda \tilde{\phi}^{l} \phi_{0} I \tilde{\psi}^{l-1} \\
&+\lambda \tilde{\phi}^{l} \phi_{0} I \psi_{0}, \\
& \mathscr{D}_{-} \mathscr{R}_{D}\left(\phi_{0}, \beta \psi_{0}\right)=\mathscr{R}_{D}\left(\mathscr{D}_{-} \phi_{0}, \beta \psi_{0}\right)-i M \mathscr{R}_{D}\left(\phi_{0}, \beta \psi_{0}\right) .
\end{aligned}
$$

From these identities, we can express $F_{ \pm}^{l}$ as follows:

$$
F_{ \pm}^{l}=\mp \frac{i}{2} \sum_{j=1}^{3} F_{j}^{l}+\text { “remainder" for } l \geq 1 \text {, }
$$

where

$$
F_{1}^{l} \equiv\left(\mathscr{D}_{-} \tilde{\phi}^{l}\right) \beta \widetilde{\psi}^{l}, \quad F_{2}^{l} \equiv\left(\mathscr{D}_{-} \phi_{0}\right) \beta \widetilde{\psi}^{l}+\left(\mathscr{D}_{-} \tilde{\phi}^{l}\right) \beta \psi_{0},
$$

$$
F_{3}^{l} \equiv 4 i \widetilde{M} \mathscr{R}_{D}\left(\mathscr{D}_{-} \phi_{0}, \beta \psi_{0}\right)
$$

Here, we note that "remainder" (given by (78)) can be handled in the same manner as $F_{j}^{l}(j=1,2$, or 3$)$. Thus, we will omit the estimate of them. We also decompose $G_{ \pm}^{l}$ as $G_{ \pm}^{l}=$ $\mp(i / 2) \sum_{j=1}^{3} G_{j}^{l}$, where

$$
\begin{gathered}
G_{1}^{l}=\left(\widetilde{\psi}^{l}\right)^{*} \beta \widetilde{\psi}^{l}, \quad G_{2}^{l}=\left(\widetilde{\psi}^{l}\right)^{*} \beta \psi_{0}+\psi_{0}^{*} \beta \widetilde{\psi}^{l}, \\
G_{3}^{l}=4 \widetilde{\mathscr{M}} \mathscr{R}_{K}\left(\psi_{0}^{*}, \beta \psi_{0}\right) .
\end{gathered}
$$

Taking $L_{t}^{4} L_{x}^{4}$-norm and $L_{t}^{\infty} H^{1 / 2}$-norm of (71) and applying Lemma 6 with $(q, r, \gamma)=(4,4,1 / 2)$ and $(2, \infty, 0)$, we have

$$
\begin{aligned}
& \left\|v^{l+1}\right\|_{L_{t}^{4} L_{x}^{4}}+\left\|v^{l+1}\right\|_{L_{t}^{\infty} H^{1 / 2}} \\
& \leq\left\|F_{1}^{l}\right\|_{L_{t}^{4 / 3} L_{x}^{4 / 3}}+\left\|G_{1}^{l}\right\|_{L_{t}^{4 / 3} H_{4 / 3}^{1 / 2}} \\
& \quad+\sum_{j=2,3}\left(\left\|F_{j}^{l}\right\|_{L_{t}^{1} H^{-1 / 2}}+\left\|G_{j}^{l}\right\|_{L_{t}^{1} L_{x}^{2}}\right) .
\end{aligned}
$$

Moreover, we remember that $\left(\widetilde{\phi}, \widetilde{\psi}^{l}\right)$ is expressed as (65).

Now, we will estimate $F_{1}^{l}$. By the Hölder inequality, we have

$$
\begin{aligned}
\left\|\left(\mathscr{D} \mathscr{B} \Phi^{l}\right) \mathscr{B} \Psi^{l}\right\|_{L_{t}^{4 / 3} L_{x}^{4 / 3}} & \lesssim\|\| \mathscr{B} \Phi^{l}(s)\left\|_{H^{1}}\right\| \mathscr{B} \Psi^{l}(s)\left\|_{L_{x}^{4}}\right\|_{L_{t}^{4 / 3}} \\
& \lesssim A\left\|s^{-\mu}\right\| \mathscr{B} \Psi^{l}(s)\left\|_{L_{x}^{4}}\right\|_{L_{t}^{4 / 3}} \\
& \leq A\left\|\mathscr{B} \Psi^{l}\right\|_{L_{t}^{4} L_{x}^{4}}\left\|s^{-\mu}\right\|_{L_{t}^{2}(I)} \\
& \lesssim A^{2} t^{1 / 2-2 \mu},
\end{aligned}
$$

for any $t \geq T$ since $v^{k} \in \mathbf{X}_{T, A}$ for $0 \leq k \leq l$. By the Hölder inequality and Remark 5 with $p=8$, we obtain

$$
\left\|\tilde{f}_{D}(s)\right\|_{L^{4}} \lesssim\left\|\phi_{0}(s)\right\|_{H_{8}^{2}}\left\|\psi_{0}(s)\right\|_{H_{8}^{1}} \lesssim A^{2} s^{-3 / 2},
$$

for any $s \geq t$. In the same manner as the proof of the estimate (83), we also obtain

$$
\left\|\left(\mathscr{D} \mathscr{B} \Phi^{l}\right) \tilde{f}_{D}\right\|_{L_{t}^{4 / 3} L_{x}^{4 / 3}} \lesssim A^{3} t^{-3 / 4-\mu},
$$

for all $t \geq T$, due to $v^{k} \in \mathbf{X}_{T, A}$ for $0 \leq k \leq l$ and (84). By the Hölder inequality and Remark 5 with $p=8 / 3,8$, we obtain

$$
\begin{aligned}
\left\|\mathscr{D} \tilde{f}_{K}(s)\right\|_{L^{2}} & \leqslant\left\|\psi_{0}(s)\right\|_{H_{8 / 3}^{2}}\left\|\psi_{0}(s)\right\|_{H_{8}^{1}} \\
& \lesssim s^{-1}\left\|\psi^{+}\right\|_{H_{8 / 5}^{5 / 2}}\left\|\psi^{+}\right\|_{H_{8 / 7}^{5 / 2}} \lesssim A^{2} s^{-1}
\end{aligned}
$$


for any $s \geq t$, where we have used properties (1) of $\alpha, \beta$, and $\mathscr{D}_{+} \psi_{0}=0$. Thus, in the same manner as the proof of the estimate (83), we obtain

$$
\left\|\left(\mathscr{D} \tilde{f}_{K}\right) \mathscr{B} \Psi^{l}\right\|_{L_{t}^{4 / 3} L_{x}^{4 / 3}} \lesssim A^{3} t^{-1 / 2-\mu},
$$

for all $t \geq T$ due to $v^{l} \in \mathbf{X}_{T, A}$ and (86). By the Hölder inequality and estimates (84) and (86), we get

$$
\begin{aligned}
& \left\|\left(\mathscr{D} \tilde{f}_{K}\right) \tilde{f}_{D}\right\|_{L_{t}^{4 / 3} L_{x}^{4 / 3}} \\
& \quad \leq\|\| \mathscr{D} \tilde{f}_{K}(s)\left\|_{L_{x}^{2}}\right\| \tilde{f}_{D}(s)\left\|_{L_{x}^{4}}\right\|_{L_{t}^{4 / 3}(I)} \lesssim A^{4} t^{-7 / 4},
\end{aligned}
$$

for all $t \geq T$. Thus by combining (83), (85), and (87)-(88), we obtain

$$
\left\|F_{1}^{l}\right\|_{L_{t}^{4 / 3} L_{x}^{4 / 3}} \lesssim A^{2} t^{1 / 2-2 \mu}
$$

for $t \geq T \geq 1$ since $\mu<1$. Next, we consider $F_{2}^{l}$. We have

$$
\left\|F_{2}^{l}\right\|_{L_{t}^{1} H^{-1 / 2}} \leq\left\|\left(\mathscr{D} \tilde{\phi}^{l}\right) \psi_{0}\right\|_{L_{t}^{1} L_{x}^{2}}+\left\|\left(\mathscr{D} \phi_{0}\right) \tilde{\psi}^{l}\right\|_{L_{t}^{1} L_{x}^{2}} \text {. }
$$

By Corollary 4 with $p=\infty$, we have

$$
\begin{aligned}
& \left\|\left(\mathscr{D} \mathscr{B} \Phi^{l}\right) \psi_{0}\right\|_{L_{t}^{1} L_{x}^{2}} \\
& \quad \leq\|\| \mathscr{B} \Phi^{l}(s)\left\|_{H^{1}}\right\| \psi_{0}(s)\left\|_{L_{x}^{\infty}}\right\|_{L_{t}^{1}} \lesssim \rho A t^{-\mu},
\end{aligned}
$$

for all $t \geq T$ since $v^{k} \in \mathbf{X}_{T, A}$ for $0 \leq k \leq l$. In the same manner as the estimate (91), we get

$$
\begin{aligned}
\left\|\left(\mathscr{D} \tilde{f}_{K}\right) \psi_{0}\right\|_{L_{t}^{1} L_{x}^{2}} & \leqslant\|\| \mathscr{D} \tilde{f}_{K}(s)\left\|_{L_{x}^{2}}\right\| \psi_{0}(s)\left\|_{L_{x}^{\infty}}\right\|_{L_{t}^{1}} \\
& \leq \rho A^{2} t^{-1},
\end{aligned}
$$

for any $t \geq T$, where we have used the estimate (86). Moreover, we also have

$$
\begin{aligned}
\left\|\left(\mathscr{D} \phi_{0}\right) \mathscr{B} \Psi^{l}\right\|_{L_{t}^{1} L_{x}^{2}} & \lesssim\|\| \phi_{0}(s)\left\|_{H_{\infty}^{1}}\right\| \mathscr{B} \Psi^{l}(s)\left\|_{L_{x}^{2}}\right\|_{L_{t}^{1}} \\
& \leq A \rho t^{-\mu},
\end{aligned}
$$

for all $t \geq T$ since $v^{l} \in \mathbf{X}_{T, A}$. In the same proof as the estimate (84), by the Hölder inequality and Remark 5 with $p=4$, we get

$$
\left\|\tilde{f}_{D}(s)\right\|_{L^{2}} \lesssim\left\|\phi_{0}(s)\right\|_{H_{4}^{2}}\left\|\psi_{0}(s)\right\|_{H_{4}^{1}} \lesssim A^{2} s^{-1},
$$

for any $s \geq t$. By estimate (94) and Corollary 4 with $p=\infty$, we have

$$
\left\|\left(\mathscr{D} \phi_{0}\right) \tilde{f}_{D}\right\|_{L_{t}^{1} L_{x}^{2}} \lesssim\|\| \phi_{0}(s)\left\|_{H_{\infty}^{1}}\right\| \tilde{f}_{D}(s)\left\|_{L_{x}^{2}}\right\|_{L_{t}^{1}} \lesssim \rho A^{2} t^{-1},
$$

for all $t \geq T$. Therefore, by combining estimates (90)-(93) and (95), we obtain

$$
\left\|F_{2}^{l}\right\|_{L_{t}^{1} H^{-1 / 2}} \lesssim \rho A t^{-\mu}
$$

for any $t \geq T \geq 1$ since $\mu<1$. Next, we consider $F_{3}^{l}$. By the definition of $\mathscr{R}_{D}$, we have

$$
\left\|\mathscr{R}_{D}\left(\mathscr{D} \phi_{0}, \psi_{0}\right)\right\|_{L_{t}^{1} H^{-1 / 2}} \lesssim \sum_{j=1,2}\left\|\mathscr{R}\left(\mathscr{D} \phi_{0}, \psi_{0, j}\right)\right\|_{L_{t}^{1} L_{x}^{2}},
$$

where we put $\psi_{0}=\left(\psi_{0,1}, \psi_{0,2}\right)^{t}$. By Lemma 8 , we can express $\mathscr{R}$ as

$$
\mathscr{R}\left(\mathscr{D} \phi_{0}, \psi_{0, j}\right) \equiv s^{-1} Z_{1}+s^{-2} Z_{2},
$$

for $s \in \mathbb{R} \backslash\{0\}$, where

$$
\begin{aligned}
Z_{1} \equiv & \left(\partial_{0} \partial_{t} \mathscr{D} \phi_{0}\right)\left(\mathscr{Z}_{1} \partial_{1} \psi_{0, j}\right) \\
& -\left(\mathscr{Z}_{1} \partial_{t} \mathscr{D} \phi_{0}\right)\left(\partial_{0} \partial_{1} \psi_{0, j}\right)+\text { similar, } \\
Z_{2} \equiv & -\left(\mathscr{Z}_{1} \partial_{2} \psi_{0, j}\right)\left(\mathscr{Z}_{2} \partial_{1} \mathscr{D} \phi_{0}\right) \\
& +\left(\mathscr{Z}_{1} \partial_{1} \mathscr{D} \phi_{0}\right)\left(\mathscr{Z}_{2} \partial_{2} \psi_{0, j}\right)+\text { similar. }
\end{aligned}
$$

By applying the Hölder inequality, we have

$$
\begin{aligned}
\left\|s^{-1} Z_{1}\right\|_{L_{t}^{1} L_{x}^{2}} \lesssim \int_{t}^{\infty} s^{-1}( & \left\|\phi_{0}\right\|_{H_{q}^{3}}\left\|\mathscr{Z} \psi_{0}\right\|_{H_{2 q /(q-2)}^{1}} \\
& \left.+\left\|\psi_{0}\right\|_{H_{q}^{2}}\left\|\mathscr{E} \phi_{0}\right\|_{H_{2 q /(q-2)}^{2}}\right) d s .
\end{aligned}
$$

By Corollary 4 with $p=q$, we get

$$
\begin{gathered}
\left\|\phi_{0}(s)\right\|_{H_{q}^{3}} \lesssim s^{-1+2 / q}\left\|\left(\langle\nabla\rangle \phi_{1}^{+}, \phi_{2}^{+}\right)\right\|_{H_{q /(q-1)}^{4-4 / q}} \lesssim A s^{-1+2 / q}, \\
\left\|\psi_{0}(s)\right\|_{H_{q}^{2}} \lesssim s^{-1+2 / q}\left\|\psi^{+}\right\|_{H_{q /(q-1)}^{4-4 / q}} \lesssim A s^{-1+2 / q},
\end{gathered}
$$

for any $s \geq t$. On the other hand, note that the commutation relations (26) hold. By applying the Sobolev inequality and the charge and energy conservation laws, we obtain

$$
\begin{aligned}
\left\|\mathscr{E} \psi_{0}\right\|_{H_{2 q /(q-2)}^{1}} & \lesssim\left\|\mathscr{E} \psi_{0}\right\|_{H^{1+2 / q}} \\
& \lesssim\left\|\mathscr{E} \psi_{0}\right\|_{H^{3 / 2}} \lesssim\left\|\left(\mathscr{E} \psi_{0}\right)(0)\right\|_{H^{3 / 2}} \lesssim A, \\
\left\|\mathscr{E} \phi_{0}\right\|_{H_{2 q /(q-2)}^{2}} & \lesssim\left\|\mathscr{E} \phi_{0}\right\|_{H^{2+2 / q}} \\
& \lesssim\left\|\mathscr{E} \phi_{0}\right\|_{H^{5 / 2}} \lesssim\left\|\left(\mathscr{E} \phi_{0}\right)(0)\right\|_{H^{5 / 2}} \lesssim A,
\end{aligned}
$$

since $q>4$. Thus, by combining (100)-(102), we get

$$
\left\|s^{-1} Z_{1}\right\|_{L_{t}^{1} L_{x}^{2}} \lesssim A^{2} t^{-1+2 / q},
$$

for any $t \geq T$. By the Hölder inequality, we have

$$
\begin{aligned}
\left\|s^{-2} Z_{2}\right\|_{L_{t}^{1} L_{x}^{2}} & \lesssim \int_{t}^{\infty} s^{-2}\left\|\mathscr{Z} \psi_{0}(s)\right\|_{H_{4}^{1}}\left\|\mathscr{Z} \phi_{0}(s)\right\|_{H_{4}^{2}} d s \\
& \lesssim A^{2} t^{-1}
\end{aligned}
$$

since in the same manner as the proof of estimates (102), we obtain

$$
\left\|\mathscr{Z} \psi_{0}(s)\right\|_{H_{4}^{1}}+\left\|\mathscr{Z} \phi_{0}(s)\right\|_{H_{4}^{2}} \lesssim A,
$$


for any $s \geq t$. Therefore, combining (97)-(98), (103), and (104), we have

$$
\left\|F_{3}^{l}\right\|_{L_{t}^{1} H^{-1 / 2}} \lesssim A^{2} t^{-1+2 / q},
$$

for all $t \geq T \geq 1$ since $q>4$.

Next, we will estimate $G_{1}^{\mathrm{l}}$. By the Leibniz formula (25) with $\kappa=1 / 2, p=4 / 3, q_{1}=q_{2}=2$, and $r_{1}=r_{2}=4$ and the Hölder inequality, we obtain

$$
\begin{aligned}
\left\|\left(\mathscr{B} \Psi^{l}\right)^{*} \mathscr{B} \Psi^{l}\right\|_{L_{t}^{4 / 3} H_{4 / 3}^{1 / 2}} & \leqslant\left\|\mathscr{B} \Psi^{l}(s)\right\|_{H^{1 / 2}}\left\|\mathscr{B} \Psi^{l}(s)\right\|_{L_{x}^{4}} \|_{L_{t}^{4 / 3}} \\
& \lesssim A\left\|s^{-\mu}\right\| \mathscr{B} \Psi^{l}(s)\left\|_{L_{x}^{4}}\right\|_{L_{t}^{4 / 3}} \\
& \lesssim A\left\|s^{-\mu}\right\|_{L_{t}^{2}(I)}\left\|\mathscr{B} \Psi^{l}\right\|_{L_{t}^{4} L_{x}^{4}} \\
& \lesssim A^{2} t^{1 / 2-2 \mu}
\end{aligned}
$$

for any $t \geq T$ since $v^{l} \in \mathbf{X}_{T, A}$. By the fractional Leibniz rule (25) again and Remark 5 with $p=4$, we have

$$
\left\|\tilde{f}_{D}(s)\right\|_{H^{1 / 2}} \lesssim\left\|\phi_{0}(s)\right\|_{H_{4}^{5 / 2}}\left\|\psi_{0}(s)\right\|_{H_{4}^{3 / 2}} \lesssim A^{2} s^{-3 / 2},
$$

for any $s \geq t$. In the same manner as the proof of the estimate (107), we obtain

$$
\begin{aligned}
\left\|\left(\mathscr{B} \Psi^{l}\right)^{*} \tilde{f}_{D}\right\|_{L_{t}^{4 / 3} H_{4 / 3}^{1 / 2}} & \lesssim\|\| \mathscr{B} \Psi^{l}(s)\left\|_{H^{1 / 2}}\right\| \tilde{f}_{D}(s)\left\|_{H^{1 / 2}}\right\|_{L_{t}^{4 / 3}} \\
& \lesssim A^{3} t^{-3 / 4-\mu},
\end{aligned}
$$

for any $t \geq T$ due to $v^{l} \in \mathbf{X}_{T, A}$ and (108). In the same manner as the proof of the estimate (109), we get

$$
\left\|\left(\tilde{f}_{D}\right)^{*} \tilde{f}_{D}\right\|_{L_{t}^{4 / 3} H_{4 / 3}^{1 / 2}} \lesssim A^{4} t^{-7 / 4}
$$

for all $t \geq T$. Thus, by combining the estimates (107) and (109)-(110), we obtain

$$
\left\|G_{1}^{l}\right\|_{L_{t}^{4 / 3} H_{4 / 3}^{1 / 2}} \lesssim A^{2} t^{1 / 2-2 \mu},
$$

for $t \geq T \geq 1$ since $\mu<1$. In the same manner as the proof of the estimates (96) and (106), we obtain

$$
\left\|G_{2}^{l}\right\|_{L_{t}^{1} L_{x}^{2}} \lesssim \rho A t^{-\mu}, \quad\left\|G_{3}^{l}\right\|_{L_{t}^{1} L_{x}^{2}} \lesssim A^{2} t^{-1+2 / q},
$$

for any $t \geq T$. Finally, by combining (82), (89), (96), (106), and (111)-(112), we obtain

$$
\left\|v^{l+1}\right\|_{\mathbf{X}_{T}} \lesssim A\left(A T^{1 / 2-\mu}+\rho+A T^{-1+\mu+2 / q}\right),
$$

for $T \geq 1$. By the estimate (113) and $1 / 2<\mu<1-2 / q$, there exist a large $T>0$ and a small $\rho>0$ such that $v^{l+1} \in \mathbf{X}_{T, A}$. In the same manner as the proof of (113), we can prove the estimate

$$
\left\|v^{l+1}-v^{l}\right\|_{\mathbf{X}_{T}} \leq \frac{1}{2}\left\|v^{l}-v^{l-1}\right\|_{\mathbf{X}_{T}},
$$

for $l \geq 1$ if $T>1$ is sufficiently large and $\rho>0$ is sufficiently small, which implies that $\left\{v^{l}\right\}_{l \geq 0}$ is a Cauchy sequence in $\mathbf{X}_{T, A}$. Theorem 1 is proved.

\section{Appendix}

In this section, we give a proof of Lemma 15. First, we prepare the following.

Lemma 16 (see [10]). Let $\kappa \in \mathbb{R}$ and let $\psi^{+}=\psi^{+}(x)$ be a $\mathbb{C}^{2}$ valued given function. Then, for any $\mathbf{C}^{2}$-valued function $\psi=$ $\psi(t, x)$, the equivalency

$$
\left\|\psi(t)-\mathscr{V}_{D}(t) \psi^{+}\right\|_{H^{\kappa}} \simeq \sum_{ \pm}\left\|\mathscr{A}_{ \pm}^{D} \psi(t)-\mathscr{U}_{ \pm, M}(t) \mathscr{A}_{ \pm}^{D} \psi^{+}\right\|_{H^{\kappa}}
$$

holds for all $t \in \mathbb{R}$.

For the proof of the lemma, see [10].

By the lemma and a decay property of $\widetilde{f}_{D}$ given by (42), we also have the following.

Corollary 17. Let $\left(\psi^{+},\left(\langle\nabla\rangle \phi_{1}^{+}, \phi_{2}^{+}\right)\right) \in\left(H^{5 / 2,1}\right)^{4}$. The final state condition (8) with $\mathbf{X}=H^{1 / 2}$ holds if and only if the identity

$$
\lim _{t \rightarrow \infty} \sum_{ \pm}\left\|\mathscr{A}_{ \pm}^{D} \Psi(t)\right\|_{H^{\kappa}}=0
$$

is valid, where $\Psi$ is defined by (41).

Before proving the corollary, we remember some properties of the operators $\mathscr{A}_{ \pm}^{D}$ given by (17) (see [10] in detail). We note that the identity

$$
(\alpha \cdot \nabla+i M \beta)^{2}=-\langle\nabla\rangle_{M}^{2} I
$$

holds due to properties (1) of Dirac matrices. Hence, by a direct calculation, we get the following identities:

$$
\mathscr{A}_{ \pm}^{D} \mathscr{A}_{\mp}^{D}=O, \quad \sum_{ \pm} \mathscr{A}_{ \pm}^{D}=I, \quad\left(\mathscr{A}_{ \pm}^{D}\right)^{2}=\mathscr{A}_{ \pm}^{D} .
$$

We put $B=\left\|\left(\psi^{+},\left(\langle\nabla\rangle \phi_{1}^{+}, \phi_{2}^{+}\right)\right)\right\|_{H^{5 / 2,1}}$.

Proof. By Lemma 16, we see that (8) with $\mathbf{X}=H^{1 / 2}$ is equivalent to

$$
\lim _{t \rightarrow \infty} \sum_{ \pm}\left\|\mathscr{A}_{ \pm}^{D} \psi(t)-\mathscr{U}_{ \pm, M}(t) \mathscr{A}_{ \pm}^{D} \psi^{+}\right\|_{H^{1 / 2}}=0
$$

By decomposition (16) and identities (A.4), we have

$$
\left\|\mathscr{A}_{ \pm}^{D} \Psi(t)\right\|_{H^{1 / 2}}=\left\|\mathscr{A}_{ \pm}^{D} \psi(t)-\mathscr{U}_{ \pm, M}(t) \mathscr{A}_{ \pm}^{D} \psi^{+}-\mathscr{A}_{ \pm}^{D} \tilde{f}_{D}\right\|_{H^{1 / 2}} .
$$

By estimate (18), the fractional Leibniz rule (25) with $p=2$ and $q_{i}=r_{i}=4(i=1,2)$, and Remark 5 with $p=4$, we get

$$
\left\|\mathscr{A}_{ \pm}^{D} \tilde{f}_{D}\right\|_{H^{1 / 2}} \lesssim\left\|\phi_{0}\right\|_{H_{4}^{5 / 2}}\left\|\psi_{0}\right\|_{H_{4}^{3 / 2}} \lesssim t^{-1} B^{2}
$$

for all $t>0$, which completes the proof of the corollary. 
Next we will prove Lemma 15.

Proof of Lemma 15. First we prove the Dirac part. By Corollary 17, we see that (52) is equivalent to

$$
\lim _{t \rightarrow \infty} \sum_{ \pm}\left\|\mathscr{A}_{ \pm}^{D} \Psi^{l}(t)\right\|_{H^{1 / 2}}=0 \quad \text { for } l \geq 0
$$

Note that the identity

$$
\mathscr{A}_{ \pm}^{D}-\mathscr{B}_{ \pm}^{D}= \pm \frac{i}{2}\langle\nabla\rangle_{M}^{-1} \mathscr{D}_{+}
$$

holds. From the Dirac part of (55), we have

$$
\mathscr{B}_{ \pm}^{D} \Psi^{l+1}=\mathscr{A}_{ \pm}^{D} \Psi^{l+1}-\langle\nabla\rangle_{M}^{-1} F^{l} \quad \text { for } l \geq 0
$$

Thus, it is sufficient to show that

$$
\lim _{t \rightarrow \infty}\left\|F^{l}\right\|_{H^{-1 / 2}}=0 \quad \text { for } l \geq 0
$$

By the Sobolev inequality and the Hölder inequality, we have, for $l \geq 1$,

$$
\begin{aligned}
\left\|F^{l}\right\|_{H^{-1 / 2}} \lesssim & \left\|\tilde{\phi}^{l}\right\|_{H^{1 / 2}}\left\|\widetilde{\psi}^{l}\right\|_{H^{1 / 2}}+\left\|\tilde{\phi}^{l}\right\|_{H^{1 / 2}}\left\|\psi^{+}\right\|_{H^{1 / 2}} \\
& +\left(\left\|\phi_{1}^{+}\right\|_{H^{1 / 2}}+\left\|\phi_{2}^{+}\right\|_{H^{-1 / 2}}\right)\left\|\widetilde{\psi}^{l}\right\|_{H^{1 / 2}} \\
& +\left\|\phi_{0}\right\|_{H_{8}^{2}}\left\|\psi_{0}\right\|_{H_{8 / 3}^{2}} \cdot
\end{aligned}
$$

By Remark 5 with $p=8,8 / 3$, we get

$$
\left\|\phi_{0}\right\|_{H_{8}^{2}} \lesssim t^{-3 / 4} B, \quad\left\|\psi_{0}\right\|_{H_{8 / 3}^{2}} \lesssim t^{-1 / 2} B \text {. }
$$

Thus, by assumptions and estimates (A.12)-(A.13), we obtain (A.11) for $l \geq 1$. In the case of $l=0$, it is easy to see (69). We omit the details. Conversely, assume (69) and will prove (52). By the decomposition $I=\sum_{ \pm} \mathscr{B}_{ \pm}^{D}$, we have only to show that

$$
\lim _{t \rightarrow \infty} \sum_{ \pm}\left\|\mathscr{B}_{ \pm}^{D} \tilde{f}_{D}\right\|_{H^{1 / 2}}=0 .
$$

We have

$$
\begin{gathered}
\left\|\mathscr{B} \tilde{f}_{D}\right\|_{H^{1 / 2}} \lesssim\left\|\mathscr{B} Q_{0}^{D}\left(\mathscr{D} \phi_{0}, \psi_{0}\right)\right\|_{H^{1 / 2}}+\text { remainder, } \\
\left\|\mathscr{B} Q_{0}^{D}\left(\mathscr{D} \phi_{0}, \psi_{0}\right)\right\|_{H^{1 / 2}} \lesssim\left\|\widehat{Q}_{0}^{D}\right\|_{H^{1 / 2}}+\left\|\partial_{t} \widehat{Q}_{0}^{D}\right\|_{H^{1 / 2}} .
\end{gathered}
$$

By the Hölder inequality and Remark 5 with $p=8,8 / 3$, we obtain

$$
\begin{aligned}
& \left\|\partial_{t} Q_{0}\left(\mathscr{D} \phi_{0}, \psi_{0, j}\right)\right\|_{H^{-1 / 2}} \\
& \quad \leq\left\|\phi_{0}\right\|_{H_{8 / 3}^{3}}\left\|\psi_{0}\right\|_{H_{8}^{1}}+\left\|\phi_{0}\right\|_{H_{8}^{2}}\left\|\psi_{0}\right\|_{H_{8 / 3}^{2}} \\
& \quad \leq t^{-1} B^{2} .
\end{aligned}
$$

Since the remainder terms in (A.15) can be estimated in the same manner as the proof of (A.17), we obtain

$$
\left\|\mathscr{B} \tilde{f}_{D}\right\|_{H^{1 / 2}} \lesssim t^{-1} B^{2},
$$

from which (A.14) follows.
Next, we consider the KG part. By the identity

$$
\|f+g\|_{H^{\kappa}}^{2}+\|f-g\|_{H^{\kappa}}^{2}=2\left(\|f\|_{H^{\kappa}}^{2}+\|g\|_{H^{\kappa}}^{2}\right),
$$

we can see that (53) is equivalent to

$$
\sum_{ \pm}\left\|\mathscr{B}_{ \pm}^{K}\left(\phi^{l}(t)-\phi_{0}(t)\right)\right\|_{H^{1}} .
$$

In the same manner as the proof of estimate (A.18), we can obtain

$$
\left\|\mathscr{B} \widetilde{f}_{K}\right\|_{H^{1}} \lesssim t^{-1} B^{2}
$$

which completes the proof of the lemma.

\section{Acknowledgment}

The author would like to express deep gratitude to an anonymous referee for the useful suggestions and comments.

\section{References}

[1] N. Bournaveas, "Low regularity solutions of the Dirac KleinGordon equations in two space dimensions," Communications in Partial Differential Equations, vol. 26, no. 7-8, pp. 1345-1366, 2001.

[2] P. D’Ancona, D. Foschi, and S. Selberg, "Local well-posedness below the charge norm for the Dirac-Klein-Gordon system in two space dimensions," Journal of Hyperbolic Differential Equations, vol. 4, no. 2, pp. 295-330, 2007.

[3] A. Grünrock and H. Pecher, "Global solutions for the DiracKlein-Gordon system in two space dimensions," Communications in Partial Differential Equations, vol. 35, no. 1, pp. 89-112, 2010.

[4] H. Pecher, "Unconditional well-posedness for the Dirac-KleinGordon system in two space dimensions," http://arxiv.org/abs/ 1001.3065.

[5] S. Selberg and A. Tesfahun, "Unconditional uniqueness in the charge class for the Dirac-Klein-Gordon equations in two space dimensions," Nonlinear Differential Equations and Applications, vol. 20, no. 3, pp. 1055-1063, 2013.

[6] A. Bachelot, "Problème de Cauchy global pour des systèmes de Dirac-Klein-Gordon," Annales de l'Institut Henri Poincaré, vol. 48, no. 4, pp. 387-422, 1988.

[7] R. B. E. Wibowo, "Scattering problem for a system of nonlinear Klein-Gordon equations related to Dirac-Klein-Gordon equations," Nonlinear Analysis: Theory, Methods \& Applications, vol. 71, no. 3-4, pp. 881-890, 2009.

[8] H. Sunagawa, "On global small amplitude solutions to systems of cubic nonlinear Klein-Gordon equations with different mass terms in one space dimension," Journal of Differential Equations, vol. 192, no. 2, pp. 308-325, 2003.

[9] Y. Kawahara and H. Sunagawa, "Global small amplitude solutions for two-dimensional nonlinear Klein-Gordon systems in the presence of mass resonance," Journal of Differential Equations, vol. 251, no. 9, pp. 2549-2567, 2011.

[10] N. Hayashi, M. Ikeda, and P. I. Naumkin, "Wave operator for the system of the Dirac-Klein-Gordon equations," Mathematical Methods in the Applied Sciences, vol. 34, no. 8, pp. 896-910, 2011. 
[11] M. Ikeda, A. Shimomura, and H. Sunagawa, "A remark on the algebraic normal form method applied to the Dirac-KleinGordon system in two space dimensions," RIMS Kôkyûroku Bessatsu B, vol. 33, pp. 87-96, 2012.

[12] P. D’Ancona, D. Foschi, and S. Selberg, "Null structure and almost optimal local regularity for the Dirac-Klein-Gordon system," Journal of the European Mathematical Society, vol. 9, no. 4, pp. 877-899, 2007.

[13] N. Hayashi and P. I. Naumkin, "Wave operators to a quadratic nonlinear Klein-Gordon equation in two space dimensions," Nonlinear Analysis: Theory, Methods \& Applications, vol. 71, no. 9, pp. 3826-3833, 2009.

[14] B. Marshall, W. Strauss, and S. Wainger, " $L^{p}-L^{q}$ estimates for the Klein-Gordon equation," Journal de Mathématiques Pures et Appliquées, vol. 59, no. 4, pp. 417-440, 1980.

[15] K. Yajima, "Existence of solutions for Schrödinger evolution equations," Communications in Mathematical Physics, vol. 110, no. 3, pp. 415-426, 1987.

[16] T. Kato and G. Ponce, "Commutator estimates and the Euler and Navier-Stokes equations," Communications on Pure and Applied Mathematics, vol. 41, no. 7, pp. 891-907, 1988.

[17] Y. Tsutsumi, "Global solutions for the Dirac-Proca equations with small initial data in $3+1$ space time dimensions," Journal of Mathematical Analysis and Applications, vol. 278, no. 2, pp. 485-499, 2003.

[18] S. Klainerman, "The null condition and global existence to nonlinear wave equations," in Nonlinear Systems of Partial Differential Equations in Applied Mathematics, vol. 23 of Lectures in Applied Mathematics, pp. 293-326, American Mathematical Society, Providence, RI, USA, 1986.

[19] S. Katayama, "A note on global existence of solutions to nonlinear Klein-Gordon equations in one space dimension," Journal of Mathematics of Kyoto University, vol. 39, no. 2, pp. 203-213, 1999.

[20] R. Kosecki, "The unit condition and global existence for a class of nonlinear Klein-Gordon equations," Journal of Differential Equations, vol. 100, no. 2, pp. 257-268, 1992.

[21] Y. Tsutsumi, "Stability of constant equilibrium for the MaxwellHiggs equations," Funkcialaj Ekvacioj, vol. 46, no. 1, pp. 41-62, 2003. 


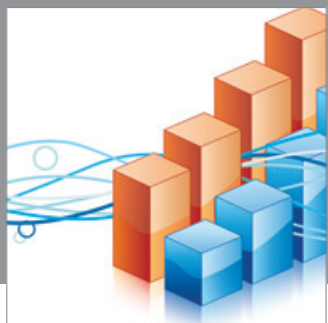

Advances in

Operations Research

mansans

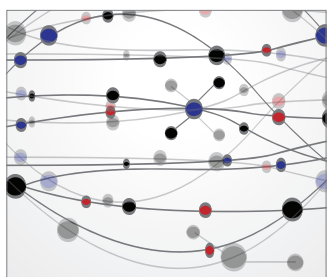

The Scientific World Journal
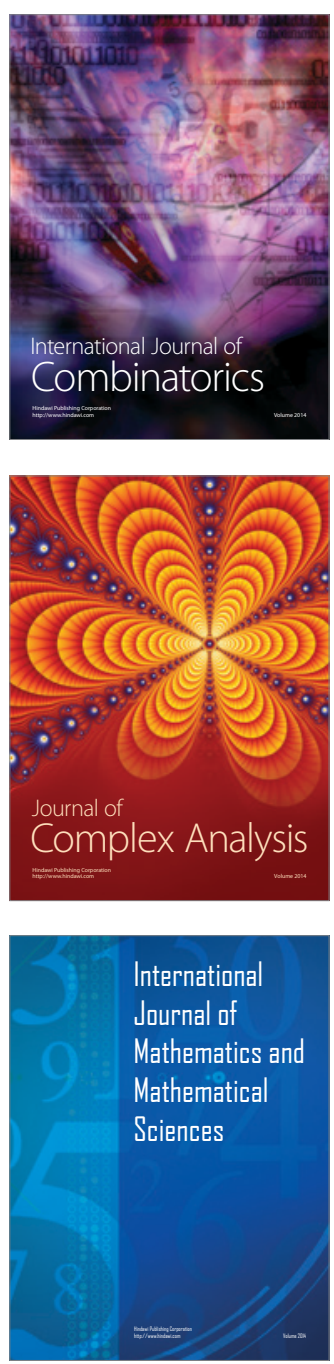
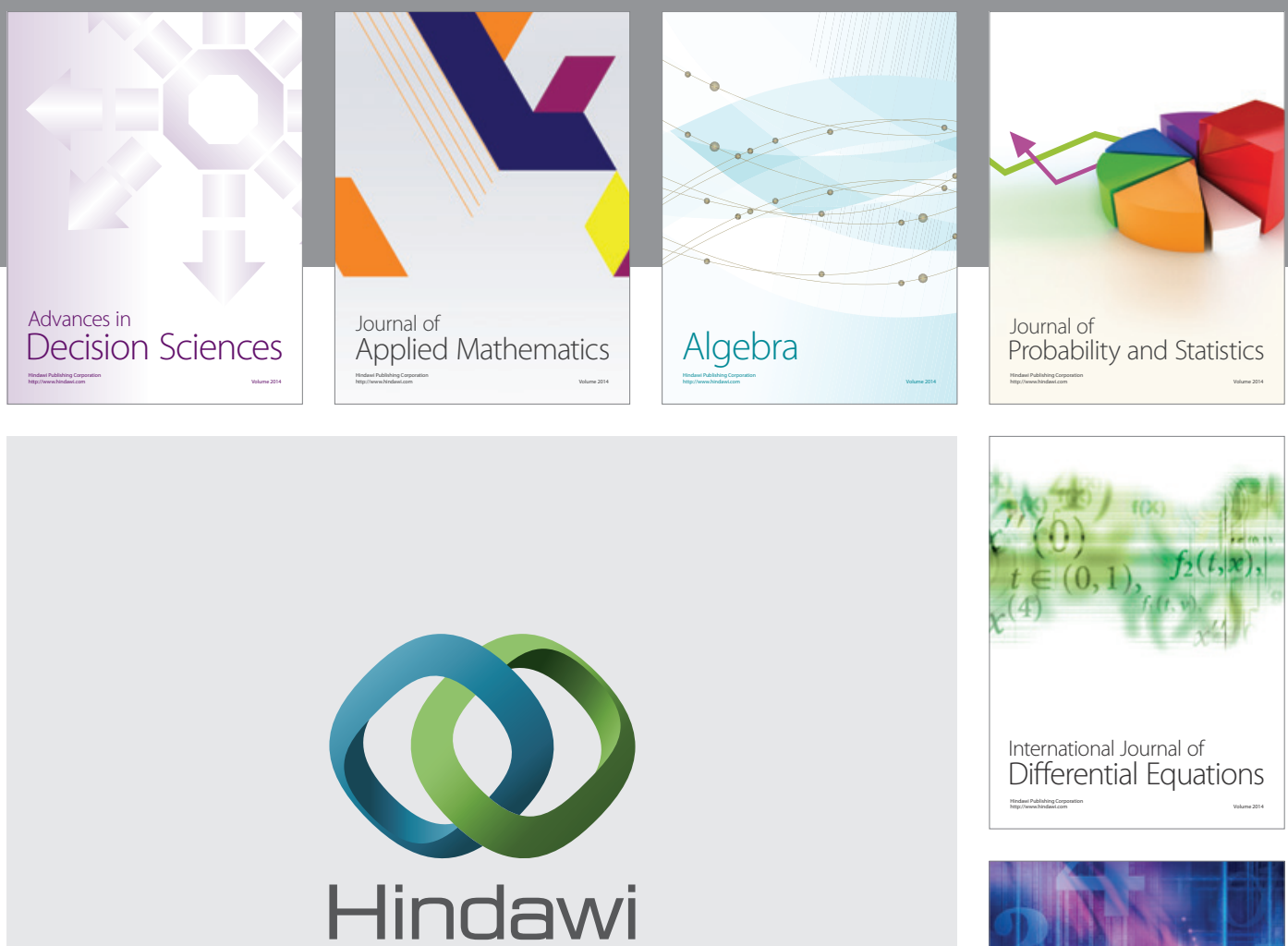

Submit your manuscripts at http://www.hindawi.com
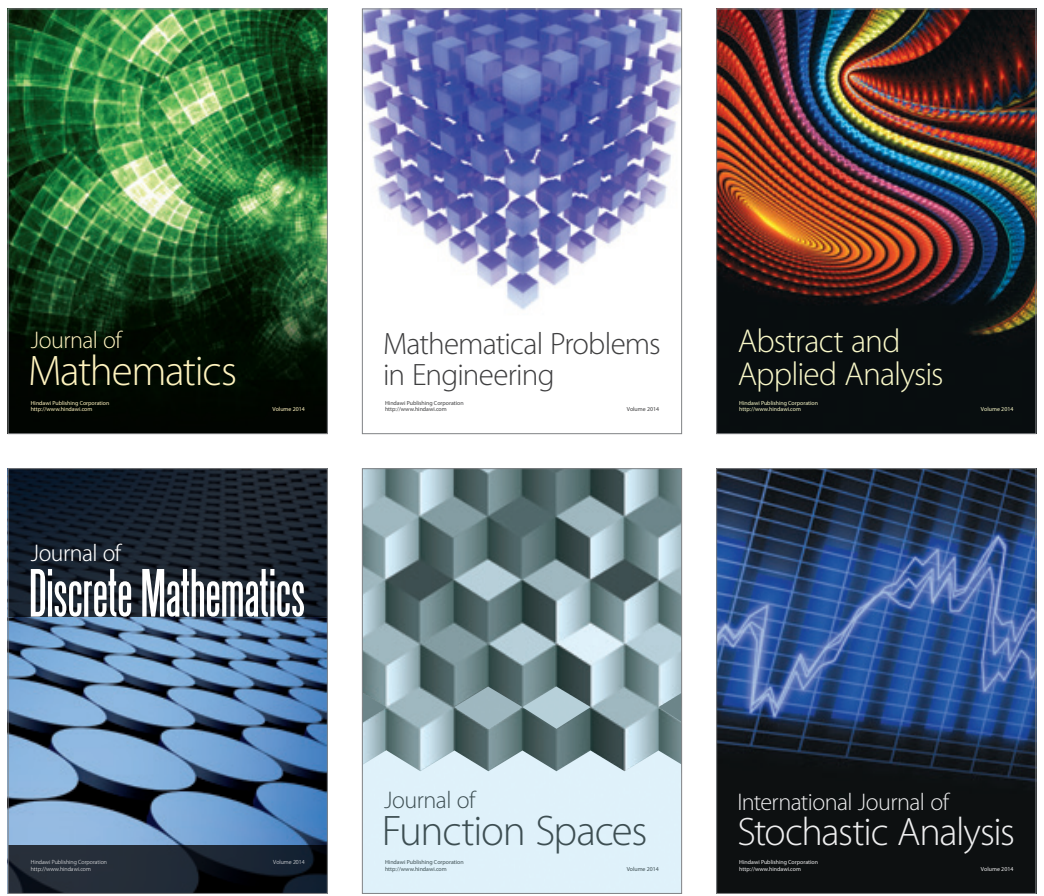

Journal of

Function Spaces

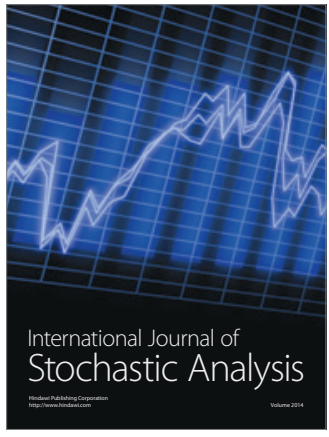

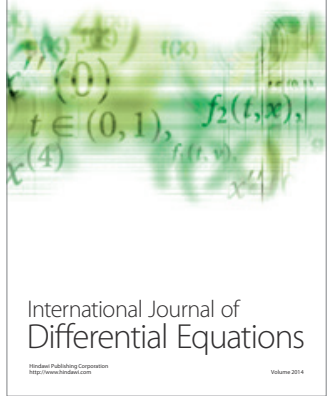
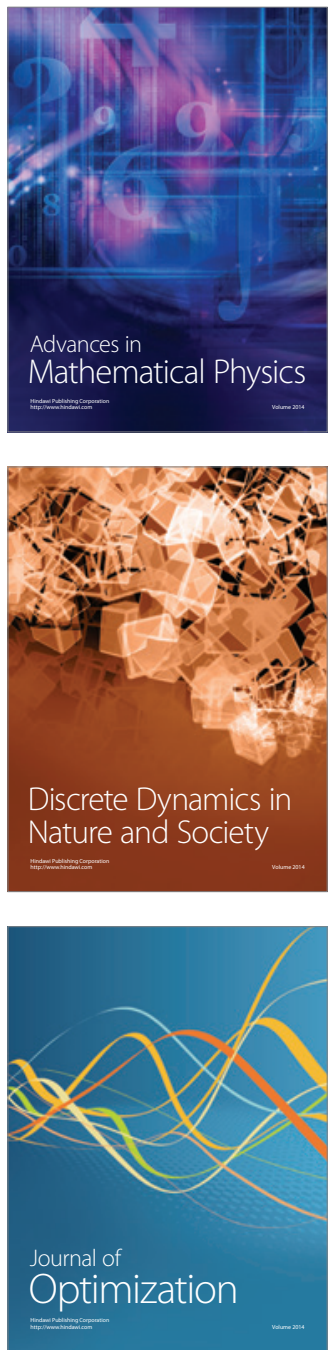